\title{
Desafios na atenção à saúde da criança durante a pandemia de COVID-19: o que não deve ser esquecido
}

\section{Challenges in child heath care during the COVID-19 pandemic: what should not be forgotten}

\author{
Ingre Paz ${ }^{1}$, Jenifer Grotto Souza ${ }^{1}$, Edna Linhares Garcia ${ }^{1}$, Suzane Frantz Krug ${ }^{1}$
}

1 - Universidade de Santa Cruz do Sul - UNISC, Santa Cruz do Sul, RS, Brasil.

ingrepaz@unisc.br

\section{RESUMO}

Introdução: um número pequeno de casos foi relatado em crianças e a compreensão da doença nesta faixa etária ainda é limitada. Objetivo: refletir sobre os principais desafios associados ao cuidado à saúde da criança durante a pandemia, de forma a não serem esquecidos cuidados essenciais ao desenvolvimento infantil como nutrição adequada e saúde mental, que permanecerão importantes mesmo após a COVID-19. Método: revisão integrativa de literatura, de caráter descritivo que teve suas buscas nas bases de dados PUBMED e SCOPUS, com os descritores Coronavirus Infections; Covid 19; Child; Breastfeeding; utilizando artigos originais completos, publicados em inglês entre os meses de março e novembro de 2020, que trataram da temática da pandemia e sua relação com cuidados à saúde das crianças. A sistematização dos dados foi realizada pela análise de conteúdo dos artigos. Resultados: dos 38 artigos encontrados, foram selecionados 17. Essa pesquisa incluiu os aspectos clínicos e epidemiológicos da COVID-19 onde observou-se mortalidade de crianças associadas à comorbidades e co-infecção com outros vírus respiratórios. Os desafios no caso de mães oligo ou assintomáticas com COVID-19 confirmada ou suspeita, os efeitos nutricionais à gestantes e crianças durante a pandemia podem estar subestimados e gerarão impactos em programas de saúde materna e infantil. Houve constatação de alterações psicossociais entre crianças e adolescentes, principalmente pela caracterização do sofrimento psíquico Conclusão: a pandemia gera uma urgência por aprender, identificar e

Palavras-chave: desenvolver recursos para enfrentar uma nova situação de crise. Devemos lembrar que há prioridades Infecção por Coronavíque jamais deveriam ser esquecidas e que já são negligenciadas há anos, como a promoção à saúde, principalmente na faixa etária pediátrica. Espera-se que este estudo traga reflexões que vão além da

Amamentação. pandemia, que impactem na forma como conduzimos o cuidado à saúde das crianças.

\begin{abstract}
Introduction: a small number of cases have been reported in children, and the understanding of how the disease affects this age group is still limited. Objective: to reflect on the main challenges of child health care during the pandemic, so not to forget essential care for child development, such as adequate nutrition and mental health, which will remain significant even after COVID-19. Method: integrative literature review, with a descriptive approach, based on searches in the PUBMED and SCOPUS databases, with the descriptors Coronavirus Infections; Covid-19; Child; and Breastfeeding. The search was conducted with complete original articles, published in English between March and November 2020, which addressed the pandemic and its relationship with child health care. The systematization of the data was carried out by analyzing the content of the articles. Results: of the 38 articles found, 17 were selected. This research included the clinical and epidemiological aspects of COVID-19, in which child mortality was associated with comorbidities and co-infection with other respiratory viruses. The challenges in the case of oligo or asymptomatic mothers with confirmed or suspected COVID-19, and the nutritional effects on pregnant women and children during the pandemic may be underestimated and impact maternal and child health programs. There was evidence of psychosocial changes among children and adolescents, mainly due to the characterization of psychological distress. Conclusion: the pandemic creates an urgent need to learn, identify, and develop resources to face a new crisis. We must remember that priorities, which have already been neglected for years, should never be forgotten, such as health promotion, especially in the pediatric age group. It is hoped that this study will bring reflections that go beyond the pandemic, impacting the way we conduct health care for children.
\end{abstract}

Keywords: Coronavirus infection Covid-19; Kids; Breast

-feeding. 


\section{INTRODUÇÃO}

Em 31 de dezembro de 2019, a Autoridade de Saúde da China alertou a Organização Mundial de Saúde (OMS) sobre uma série de casos de pneumonia de etiologia desconhecida na cidade de Wuhan, província de Hubei, região populosa da China. Em janeiro, um novo coronavírus foi identificado a partir de amostra de swab de orofaringe de um paciente. Posteriormente a doença foi denominada doença por coronavírus (COVID-19) e determinada como emergência de saúde pública de interesse internacional pela OMS. Nos meses subsequentes atingiu todos os continentes, tomando proporções mundiais. Até março de 2020, COVID-19 foi responsável por mais de 110.000 infecções e 4000 mortes em todo o mundo. ${ }^{1,2}$

Consequências da pandemia de COVID-19 sobre a saúde principalmente de crianças e adolescentes no Brasil, assim como em outros países da América Latina, tem potencial muito mais negativo do que o que vem sendo relatado em países da Europa e América do Norte. ${ }^{3}$

Um número pequeno de casos de COVID-19 foi relatado em crianças e a compreensão da doença nesta faixa etária ainda é limitada, embora observe-se que a maioria dos casos nesta faixa etária apresenta-se de forma branda e oligossintomática. ${ }^{4}$ Devido a este perfil epidêmico, em caso de mães com suspeita ou confirmação de infecção por COVID-19 no momento do parto, o alojamento conjunto e a amamentação são aconselhados. No caso de mães com sintomas graves, incapazes de prestarem os cuidados ao recém-nascido, a criança deve ser mantida em unidade separada da mãe, mas o leite materno pode ser ordenhado e oferecido à criança, não sendo necessária qualquer técnica de pasteurização. ${ }^{5}$

Considerando-se a disseminação da COVID-19 e não haver tratamento curativo eficaz, um sistema imunológico saudável é uma das ferramentas mais importantes para a manutenção da saúde. Nos primeiros 6 meses de vida, o leite humano é a melhor fonte de nutrientes para a criança, adaptando-se às necessidades calóricas e contribuindo para o fortalecimento do sistema imunológico. Após a introdução de alimentação complementar, uma dieta rica em vitaminas e oligoelementos são essenciais ao funcionamento normal do sistema imune e defesa contra infecções virais. A desnutrição aumenta a morbimortalidade na faixa etária pediátrica, resultando em aumento na taxa de infecções e atraso na recuperação de doenças, além de representar impacto econômico importante nos sistemas de saúde. ${ }^{6}$

As intervenções governamentais e institucionais no contexto da pandemia têm apresentado objetivo principal a contenção da doença e possíveis tratamentos com a finalidade de manter a saúde física e mental da população. No entanto, muitas prioridades anteriores à pandemia para manter essa saúde têm sido esquecidas. ${ }^{7}$ Às necessidades de saúde das populações continuam existindo apesar do foco estar centrado no combate à pandemia. Doenças cardiovasculares continuam gerando internações e incremento em morbidade e mortalidade. Da mesma forma, as privações nutricionais continuam afetando crianças em todas as partes do mundo, gerando sequelas a longo prazo.

Diante do exposto, o objetivo deste estudo é refletir sobre os principais desafios associados ao cuidado à saúde da criança durante a pandemia, de forma a não serem esquecidos cuidados essenciais ao desenvolvimento infantil como nutrição adequada e saúde mental, que permanecerão importantes mesmo após a COVID-19.

\section{MÉTODO}

O presente estudo trata de uma Revisão Integrativa de literatura, de estudo de caráter descritivo que consiste em fazer uma revisão rigorosa de artigos com diferentes metodologias e integrar seus resultados em torno de um objetivo.

Os seguintes critérios de inclusão foram adotados para selecionar os artigos para análise: a) estudos publicados na íntegra; b) estudos publicados no limite temporal entre março e novembro de 2020; c) estudos que abordassem os cuidados essenciais à saúde da criança durante a pandemia de COVID-19. Os critérios de exclusão adotados foram: a) pesquisas que trouxeram abordagens terapêuticas relacionadas ao manejo clínico da COVID-19; b) estudos relacionados a outras faixas etárias além da pediatria.

Com base na aplicação destes critérios, as pesquisadoras conduziram uma busca nas bases de dados Pubmed e Scopus entre os meses de agosto e novembro de 2020. Para ordenar a busca de artigos, utilizaram-se as palavras-chave contidas nos Descritores em ciências da Saúde (DeCs) 
"Coronavirus Infections"; "Covid 19"; "Child";

"breastfeeding". Seus termos sinônimos foram combinados com o uso de operadores de lógica "AND" e "OR".

\section{RESULTADOS}

A sistematização dos dados foi realizada pela análise de conteúdo dos artigos iniciando pela leitura do título nas bases de dados e então os artigos selecionados nesta fase passaram para o exame dos resumos, no qual foram aplicados critérios de elegibilidade para após, procedermos à leitura do texto na íntegra. O resultado inicial da busca resultou em 38 artigos possivelmente relevantes dentro da temática escolhida, após aplicação de critérios de inclusão e análise detalhada, permaneceram 17 estudos. O processo de seleção está representado na figura 1.

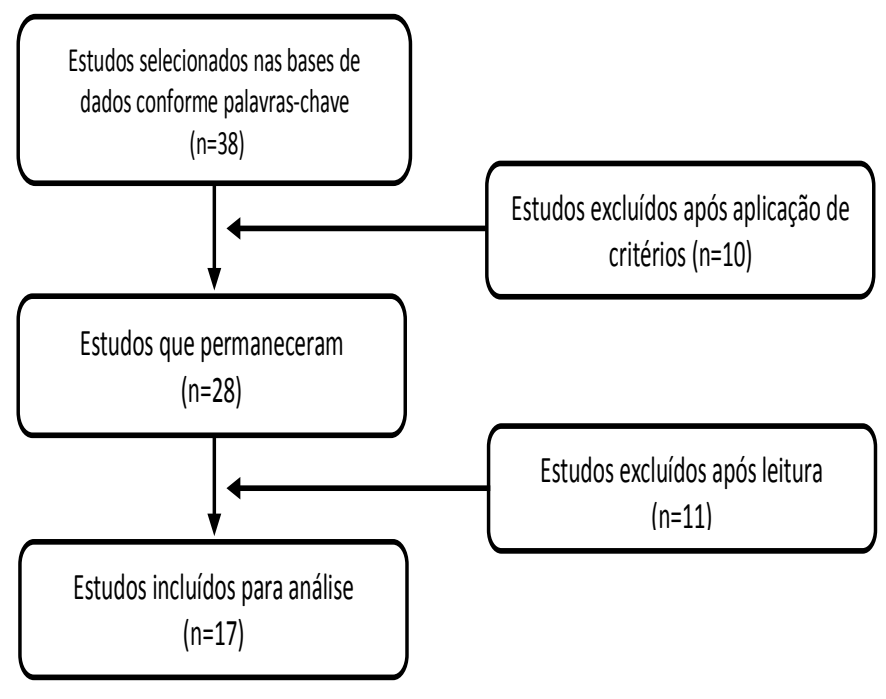

Figura 1 - Fluxograma do processo de eleição dos estudos incluídos na revisão.

Os estudos tiveram data de publicação entre junho e outubro de 2020 e foram realizados em diversos países como Estados Unidos, França e Reino Unido, sendo todas pesquisas qualitativas.

Após a análise dos artigos incluídos, a discussão foi dividida em 3 categorias temáticas sendo: A COVID-19 em crianças, aleitamento materno e COVID-19, alimentação saudável: como manter um padrão alimentar adequado das crianças diante do isolamento social, saúde mental das crianças durante à pandemia e suas repercussões.

\section{DISCUSSÃO}

\section{A Covid 19 em Crianças}

Um pequeno número de casos de COVID-19 foi descrito em crianças e a compreensão da doença nesta faixa etária é ainda limitada. Na China, em fevereiro, uma série de casos foi publicada pelo Centro Chinês de Controle e Prevenção de doenças, evidenciando que o diagnóstico entre crianças foi de $1,2 \%$, sem nenhum óbito registrado. ${ }^{8}$ Nos estudos subsequentes, observou-se mortalidade de algumas crianças, frequentemente com comorbidades associadas. Em $11-46 \%$ dos casos são encontradas co-infecções com outros vírus respiratórios como adenovírus, rinovírus e influenza. Nos estudos iniciais a respeito da doença na faixa etária pediátrica, não havia relatos de apresentações mais graves, apenas quando havia histórico de comorbidades. ${ }^{4}$

Diversas hipóteses foram elaboradas para explicar a menor incidência em crianças, estudos mais atuais destacam que os casos diagnosticados de coronavírus em crianças menores do que 18 anos é de cerca $2 \%$ do total dos casos. Um sistema imune inato mais ativo, menor exposição a cigarros e poluentes, imaturidade dos receptores da enzima conversora de angiotensina 2 (ECA-2), locais de ligação para a entrada do coronavírus nas células podem ter relação com menor incidência. Se considerarmos que as crianças representam $20 \%$ da população total, podemos inferir que a chance de uma criança se infectar é de 1/10 do restante da população. O papel das crianças na transmissão do SARS-Cov-2 parece ser pequeno, dados sugerem que na maior parte das vezes as crianças adquirem o vírus de adultos contaminados dentro de casa e os casos de transmissão conhecidos são raros de uma criança para outra. ${ }^{9}$

Entre as manifestações clínicas relatadas em crianças nos estudos, a variedade de apresentações é ainda maior que em adultos. Cerca de $90 \%$ dos casos nesta faixa etária são assintomáticos ou com sintomas leves, que incluem tosse, febre, odinofagia, diarreia, vômitos e coriza. As manifestações gastrointestinais são quase tão frequentes quanto sintomas respiratórios, o que muitas vezes torna o diagnóstico desafiador. ${ }^{9}$ Embora manifestações brandas sejam mais comuns, observou-se que a infecção por COVID-19 foi significativa em Unidades de terapia intensiva pediátricas e que formas graves ocorriam em crianças, mesmo quando previamente hígidas, embora em frequência inferior à encontrada em adultos. As 
Tabela 1 - Publicações selecionadas para o estudo

\begin{tabular}{rll}
\hline Título e Ano & Autor (es) Objetivo
\end{tabular}

Clinical features of pediatric patients with Song, Wenliang et. al. coronavirus disease (COVID-19) (2020)

Coronavirus Infections in Children Including COVID-19 (2020) ${ }^{4}$

Zimmermann, Petra Curtis, Nigel

Breastfeeding and Coronavirus Disease-2019 Davanzo, Riccardo et. al. $(2020)^{5}$

Enhancing immunity in viral infections, with special emphasis on COVID-19: A review (2020) ${ }^{6}$

Characteristics of and Important Lessons from the Coronavirus Disease 2019 (COVID-19) $(2020)^{8}$

Coronavirus disease 2019 (COVID-19) in children: prevalence, diagnosis, clinical symptoms, and treatment $(2020)^{9}$

Characteristics and Outcomes of Children With Coronavirus Disease 2019 (COVID-19) $(2020)^{10}$

Standard and Specialized Infant Formulas in Europe: Making, Marketing, and Health Outcomes (2020) ${ }^{11}$

Breastfeeding in the 21st century: Epidemiology, Victoria, C. G. et al. mechanisms, and lifelong effect (2020) ${ }^{12}$

Evidence of a significant secretory-IgA-dominant SARS-CoV-2 immune response in human milk following recovery from COVID-19 (2020) $)^{13}$

Reconciling breast-feeding and early food introduction guidelines in the prevention and management of food allergy $(2020)^{14}$

Ministério da Saúde. Secretaria de Atenção à Saúde. Departamento de Atenção Básica. Dez passos para uma alimentação saudável: guia alimentar para crianças menores de dois anos: um guia para o profissional de saúde na atenção básica $(2015)^{15}$

Association of infant and young child feeding practices with cognitive development at 10-12 years: A birth cohort in rural Western China $(2020)^{16}$

A call to action to address COVID-19-induced global food insecurity to prevent hunger, malnutrition, and eating pathology $(2020)^{17}$

Child malnutrition and COVID-19: the time to act is now (2020) ${ }^{18}$

Mental health and psychosocial considerations during the COVID-19 outbreak (2020) ${ }^{19}$

Mitigate the effects of home confinement on children during the COVID-19 outbreak (2020) $)^{20}$
Jayawardena, R. et al.

Wu, Zunyou

McGoogan, Jennifer M

Zardini, Hadi Zare et. al.

Shekerdemian, L. S, Relatar características clínicas de recémMahmood NR, Wolfe KK, nascidos infectados por mães com et al.

Dipasquale, V. et al.

Fox, A. et al.

Netting, M. J.; Allen, K. J. COVID-19

Avaliar indicações, diferenças e composição nutricional das fórmulas infantis

Avaliar os benefícios de longo prazo do aleitamento materno na saúde de crianças

Avaliar a presença de anticorpos contra COVID-19 no leite materno de mães infectadas

Avaliar determinantes da amamentação e introdução alimentar

Brasil, Ministério da Elaborar recomendações sobre alimentação Saúde. $\quad$ saudável para a infância

Zhu, Z. et al.

Examinar a associação da amamentação e introdução alimentar com o desenvolvimento cognitivo do adolescente em países de baixa e média renda.

Paslakis, G; Dimitropoulos, Avaliar impacto da pandemia por G; Katzman, D.K.

COVID-19 na segurança alimentar

Fore, H.H. et al.

Who - World Health Organization.

Avaliar impacto da pandemia por COVID-19 na segurança alimentar

Orientar cuidados com a saúde mental e psicossocial durante a pandemia por COVID-19

Mitigar os efeitos do distanciamento social na saúde mental de crianças durante a pandemia por COVID-19 
comorbidades ainda aparecem como importante fator relacionado ao desfecho desfavorável da patologia. A resposta ainda não está clara do motivo pelo qual a reação à doença em crianças seja distinta. Sugere-se que devido a exposição a outros vírus respiratórios, comum nos primeiros anos de vida, possa produzir algum tipo de imunidade cruzada ao COVID. Também existe a possibilidade de que o sistema imune das crianças tenha interação diferente com o vírus, uma vez que adultos com maior frequência desenvolvem doença inflamatória multissistêmica. ${ }^{10}$

\section{Aleitamento Materno e Covid 19}

O leite humano é a melhor fonte de nutrientes para recém-nascidos, adaptando-se às mudanças das necessidades nutricionais decorrentes do processo de crescimento e desenvolvimento da criança. A Organização Mundial da Saúde (OMS) definiu o aleitamento materno exclusivo como alimentação padrão para os 6 primeiros meses de vida e, até os dois anos de idade, amamentação com alimentação complementar saudável. Todavia, a prevalência de amamentação permanece abaixo do desejado: na Europa, estima-se que apenas $25 \%$ das crianças tenham sido amamentadas exclusivamente até os 6 meses. ${ }^{11}$. Mundialmente, a prevalência de amamentação aos 12 meses é mais elevada em países em desenvolvimento, principalmente na África Subsaariana, sul da Ásia e América Latina. Países desenvolvidos têm os menores índices, com prevalência próximo a $20 \% .^{12}$

Além das prováveis consequências da infecção pela COVID-19 em gestantes e fetos, há uma grande preocupação com o efeito potencial da infecção no período neonatal e o manejo adequado do binômio mãe-filho, incluindo a compatibilidade da infecção materna com a amamentação. O Center for Disease Control and Prevention (CDC), órgão de vigilância do governo dos Estados Unidos, em sua Diretriz Interina sobre Amamentação, reafirma que a amamentação é importante proteção contra doenças e seu início e continuidade devem ser priorizados. No caso de mães oligo ou assintomáticas com COVID-19 confirmada ou suspeita, a amamentação direta é aconselhável desde que sob rigorosas medidas de controle de infecção. No caso de mães gravemente doentes, incapazes de cuidar do recém-nascido, o bebê deve ser mantido em unidade distinta a da mãe e o leite materno pode ser ordenhado e ofertado à criança sem necessidade de pasteurização, uma vez que não há evidência robusta que estabeleça nexo causal entre a transmissão do coronavírus e amamentação. ${ }^{5}$

No Brasil, o Ministério da saúde recomenda por meio de sua Nota Técnica, que o aleitamento seja mantido em caso de infecção pela COVID-19, desde que a mãe deseje amamentar e esteja em condições clínicas para tal. Caso a mãe não se sinta segura em amamentar enquanto estiver com a doença, recomenda-se que seu leite seja retirado e ofertado a criança. Neste documento, orienta-se a higiene das mãos pelo menos 20 segundos antes de tocar o bebê ou realizar extração do leite manualmente ou com bomba extratora, além do uso de máscara facial pela mãe cobrindo completamente boca e nariz durante as mamadas. Recomenda-se que durante a amamentação, deve se evitar falar ou tossir, e caso ocorra, a máscara deve ser imediatamente trocada. ${ }^{2}$

A literatura não expõe de forma clara a relevância dos profissionais de saúde em torno da promoção do aleitamento materno. Há poucos estudos que demonstram o papel dos profissionais de saúde que podem constituir uma equipe de apoio à amamentação, incluindo elementos de educação, resolução de problemas e suporte adequado. Ressaltase a necessidade de atualização constante da equipe que presta atendimento em especial a pacientes com suspeita ou confirmados de Covid-19 por tratar - se de uma doença nova e estar em constante mudança. Uma visão mais global e coletiva do trabalho tornase necessária para um melhor aproveitamento das qualidades dos profissionais em relação à saúde materno-infantil e principalmente ao aleitamento materno. $^{13}$

\section{Alimentação Saudável: Como Manter um Padrão Alimentar Adequado das Crianças Diante do Isolamento Social?}

Várias vitaminas, incluindo A, B, B12, C, $\mathrm{D}$ e folato, além de oligoelementos como zinco, magnésio, selênio e cobre desempenham papéis complementares no apoio a resposta imune inata e adaptativa, principalmente na faixa etária pediátrica em que a imunidade está em formação. As deficiências ou estados sub-ótimos de micronutrientes afeta negativamente a função imune e pode diminuir a resistência contra infecções. Outros nutrientes como ácidos graxos e ômega-3 também têm importante impacto nas respostas inflamatórias. Portanto, uma alimentação saudável tem estreita relação com uma resposta adequada do organismo à exposição à 
patógenos. ${ }^{6,14}$

A alimentação saudável é fundamental para a criança, principalmente nos primeiros dois anos de vida. Para promoção dessa prática, o Ministério da Saúde (MS) do Brasil segue as recomendações da Organização Mundial de Saúde (OMS) para promover a alimentação infantil saudável e prevenir distúrbios nutricionais disponibilizando aos profissionais e população ferramentas educativas com orientações alimentares, a exemplo do manual Dez Passos para uma Alimentação Saudável: guia alimentar para menores de dois anos. Esse material direciona a orientação sistemática da alimentação infantil pelos profissionais de saúde na consulta de puericultura, passíveis de adaptação à realidade de cada família. ${ }^{15}$

Recomendações globais de alimentação infantil da Organização Mundial da Saúde (OMS) e Fundo das Nações Unidas para a Infância (UNICEF) constituem estratégia global e recomendam aleitamento materno exclusivo até os 6 meses e alimentação complementar segura a partir dos 6 meses de idade. Segundo o principal documento publicado na área, a Estratégia Global para alimentação de bebês e crianças publicada pela OMS, nesta faixa etária, a necessidade energética e de nutrientes para o bebê começa a exceder o que é fornecido pelo leite materno e a criança já tem funções motoras e cognitivas que permitem a introdução de alimentos sólidos na sua alimentação. Se os alimentos forem fornecidos após esta idade ou forem inadequados, poderá haver deficiência nutricional e comprometimento do ganho pondero estatural da criança. ${ }^{14}$

Práticas adequadas de alimentação infantil nos dois primeiros anos de vida são importantes moduladores do desenvolvimento cerebral e podem resultar em efeitos positivos no desenvolvimento cognitivo. Uma dieta complementar composta por quantidades substanciais de ferro, seja pela ingestão de carnes, vegetais ou alimentos enriquecidos com ferro tem potencial para impedir ferropenia em crianças, que também tem associação com adequado desenvolvimento neuropsicomotor. ${ }^{16}$

Toda cadeia de suprimentos de alimentos incluindo a produção, seu transporte, distribuição e consumo durante a pandemia do COVID-19 tiveram bloqueios, forçando milhares de famílias a acessar alternativas pobres em nutrientes, assim alertas globais de insegurança alimentar foram emitidos, sendo associada a inadequações alimentares, déficits cognitivos, comprometimento do crescimento, problemas crônicos de saúde física e mental e morte. ${ }^{17}$,

${ }^{18}$ Acredita-se que efeitos nutricionais projetados da pandemia COVID-10 podem estar subestimados, pois não levam em conta o efeito potencial na nutrição materna relacionado ao crescimento intrauterino e deficiências de micronutrientes e impactos em programas de saúde materna e infantil. ${ }^{6,17}$

\section{Saúde Mental Das Crianças Durante à Pandemia e suas Repercussões}

Em 18 de março de 2020 a Organização Mundial de Saúde (OMS) emitiu uma nota técnica convocando os profissionais da saúde a atentaremse e organizarem-se para uma emergência sanitária, estimulando ações globais focadas no bem-estar psicossocial devido consequências potenciais na saúde mental. ${ }^{19}$ A complexidade envolvida na definição de saúde mental infantojuvenil dada à conceituação pela OMS, em livre tradução: (...) a capacidade de se alcançar e se manter um bom funcionamento psicossocial e um estado de bem-estar em níveis ótimos (...) Ela auxilia o jovem a perceber, compreender e interpretar o mundo que está a sua volta, a fim de que adaptações ou modificações sejam feitas em caso de necessidade [...]. ${ }^{19}$

É fato que as crianças estão sofrendo as consequências dessa pandemia tanto quanto os adultos, no entanto, muitas vezes, sua saúde mental é negligenciada. Estima-se que cerca de um terço de uma população exposta a um desastre, como a COVID-19, pode apresentar manifestações psicopatológicas se nenhuma intervenção psicossocial for realizada. A constatação de alterações psicossociais entre crianças e adolescentes, principalmente pela caracterização do sofrimento psíquico e questões emocionais são uma grande preocupação durante a COVID-19. Estudos demonstram processo de luto principalmente pela perda da liberdade, do convívio com amigos e escola. Algumas preocupações, alterações do humor, apetite e sono, são esperados. ${ }^{20}$

Muitos pais estão se sentindo sobrecarregados com suas atividades domésticas, com o trabalho, bem como com as novas demandas das crianças. As famílias precisaram se adaptar em casa pois estão privadas da sua habitual rede de apoio com a pandemia. A falta e/ou a mudança dessa rede resultam em restrição de interações para as crianças, intensificado o convívio no núcleo familiar. Até o momento, inquéritos populacionais vêm corroborando a 
importante premissa que embora menos susceptíveis às formas clínicas graves da COVID-19, crianças e adolescentes não são indiferentes ao seu impacto, quando considerada a dimensão mental. ${ }^{19}$

Algumas estratégiasparaprevenirrepercussões negativas na saúde mental entre as crianças precisam ser feitas, como intervenção sobre os estressores ambientais no contexto de cada um, identificando as vulnerabilidades, os fatores de risco e os de proteção. Outra sugestão é trabalhar a resiliência, capacidade de dar sentido e de transformar positivamente suas experiências de vida, mesmo que no atual momento sejam negativas. Promover saúde mental nesse grupo etário inclui adaptação ao estresse, cuidados com o sono, atividade física e convívio familiar saudável, tríade esta que, respeitando as normas de segurança, deve estar na agenda do cuidado dessa população. ${ }^{20}$

\section{CONCLUSÃO}

Essa pesquisa incluiu os aspectos clínicos e epidemiológicos da COVID-19 na infância, bem como os desafios diante de cuidados essenciais como aleitamento materno, os aspectos nutricionais frente ao isolamento social e a saúde mental. A pandemia gera uma urgência por aprender, identificar e desenvolver recursos para enfrentar uma nova situação de crise, um aprendizado fundamental para o desenvolvimento individual e coletivo do ser humano, uma estratégia essencial para a garantia da saúde física e mental. Devido ao curto intervalo de tempo que separa o início da disseminação do coronavírus na China e o momento atual em que vivemos, muitos questionamentos ainda não tem resposta: Haverá uma vacina eficaz para o combate à doença? Serão desenvolvidos tratamentos eficazes? Como será a vida das pessoas depois que esta pandemia passar? Como manter a saúde mental diante de um cenário tão adverso?

Embora não tenhamos respostas para esses questionamentos e para tantos outros, devemos lembrar que há prioridades que jamais deveriam ser esquecidas e que já são negligenciadas há anos, como a promoção à saúde, principalmente na faixa etária pediátrica. A manutenção do aleitamento materno exclusivo até os 6 meses de vida, uma alimentação saudável e balanceada, uma infância a ser vivida com atividades lúdicas e ao ar livre, hábitos de higiene adequados, são cuidados que devem ser lembrados a todo momento, independentemente de haver uma pandemia, pois impactam significativamente na vida das crianças e nos desfechos na sua saúde quando adultos. Espera-se que este estudo traga reflexões que vão além da pandemia, reflexões que impactem na forma como conduzimos o cuidado à saúde das crianças e para que as prioridades sempre sejam a promoção à saúde e não do cuidado às doenças.

\section{REFERÊNCIAS}

1. Harapan H, Itoh N, Yufika A, Winardi W, Keam S Te H, Megawati D, Hayati Z, Wagner AL, Mudatsir M. Coronavirus disease 2019 (COVID-19): A literature review. J. Infect. Public Health 2020;13(5):667-73. doi: https://dx.doi.org/10.1016/j. jiph.2020.03.019

2. Song W, Li J, Zou N, Guan W, Pan J, Xu W. Clinical features of pediatric patients with coronavirus disease (COVID-19). J Clin Virol 2020;127:104377. doi: https://dx.doi.org/10.1016/j. jcv.2020.104377

3. Secretaria de Atenção Primária à Saúde. Coordenação de Saúde da Criança e Aleitamento Materno. Nota Técnica No15/2020-COCAM/CGCIVI/DAPES/SAPS/MS [documento na internet]. Brasil: Ministério da Saúde; 2020 [atualizado em 20 de maio de 2021; citado em 10 de agosto de 2020]. Disponível em: https://www.gov.br/saude/pt-br/coronavirus/publicacoestecnicas/notas-tecnicas/nota-tecnica-n-15-2020.pdf/view

4. Zimmermann P, Curtis N. Coronavirus Infections in Children Including COVID-19. The Pediatr. Infect. Dis. J 2021;39(5):35568. doi: https://dx.doi.org/10.1097/INF.0000000000002660

5. Davanzo R, Moro G, Sandri F, Agosti M, Moretti C, Mosca

F. Breastfeeding and coronavirus disease-2019: Ad interim indications of the Italian Society of Neonatology endorsed by the Union of European Neonatal \& Perinatal Societies. Matern Child Nutr. 2020;16(3):e13010. doi: https://dx.doi.org/10.1111/ men. 13010

6. Jayawardena R, Sooriyaarachchi P, Chourdakis M, Jeewandara C, Ranasinghe P. Enhancing immunity in viral infections, with special emphasis on COVID-19: A review. Diabetes Metab Syndr. 2020;14(4):367-82. doi: https://dx.doi.org/10.1016/j. dsx.2020.04.015

7. Vieira CM, Franco OH, Restrepo CG, Abel T. COVID-19: The forgotten priorities of the pandemic. Maturitas 2020;136:38-41. doi: https://dx.doi.org/10.1016/j.maturitas.2020.04.004

8. Wu Z, McGoogan JM. Characteristics of and Important Lessons From the Coronavirus Disease 2019 (COVID-19) Outbreak in China: Summary of a Report of 72314 Cases From the Chinese Center for Disease Control and Prevention. JAMA 2020;323(13):1239-42. doi: https://dx.doi.org/10.1001/ jama.2020.2648

9. Zare H, Soltaninejad H, Ferdosian F, Hamidieh AA, Memarpoor-Yazdi M. Coronavirus disease 2019 (COVID-19) in children: prevalence, diagnosis, clinical symptoms, and treatment. Int J Gen Med 2020;13:477-82. doi: https://dx.doi. org/10.2147/IJGM.S262098

10. Shekerdemian LS, Mahmood NR, Wolfe KK, et al. Characteristics and Outcomes of Children With Coronavirus Disease 2019 (COVID-19) Infection Admitted to US and Canadian Pediatric Intensive Care Units. JAMA Pediatr 2020;174(9):868-73. doi: https://dx.doi.org/10.1001/ 
jamapediatrics.2020.1948

11. Dipasquale V, Serra G, Corsello G, Romano C. Standard and Specialized Infant Formulas in Europe: Making, Marketing, and Health Outcomes. Nutr Clin Pract. 2020;35(2):273-81. doi: https://dx.doi.org/10.1002/ncp.10261

12. Victora CG, Bahl R, Barros AJD, França GVA, Horton S, Krasevec J, Murch S, Sankar MJ, Walker N, Rollins NC. Breastfeeding in the 21st century: Epidemiology, mechanisms, and lifelong effect. Lancet 2020;387:475-90. doi: https://dx.doi. org/10.1016/S0140-6736(15)01024-7

13. Fox A, Marino J, Amanat F, Krammer F, Hahn-Holbrook J, Zolla-Pazner S, Powell RL. Evidence of a significant secretoryIgA-dominant SARS-CoV-2 immune response in human milk following recovery from COVID-19. MedRxiv 2020;(preprint). doi: https://dx.doi.org/10.1101/2020.05.04.20089995

14. Netting MJ, Allen KJ. Reconciling breast-feeding and early food introduction guidelines in the prevention and management of food allergy. J Allergy Clin Immunol 2019;144(2):397-400. doi: https://dx.doi.org/10.1016/j.jaci.2019.06.004

15. Secretaria de Atenção à Saúde. Departamento de Atenção Básica. Dez passos para uma alimentação saudável: guia alimentar para crianças menores de dois anos: um guia para o profissional de saúde na atenção básica [documento na nternet]. Brasil: Ministério da Saúde; 2015 [atualizado em 22 de junho de 2015; citado em 10 de agosto de 2020]. Disponível em: http://bvsms.saude.gov.br/bvs/publicacoes/guia_dez_passos_ alimentacao_saudavel_2ed.pdf

16. Zhu Z, Cheng Y, Qi Q, Lu Y, Ma S, Li S, Li H, Elhoumed M, Tsegaye S, Fawzi WW, Sudfeld CR, Yan H, Dibley MJ, Zeng L. Association of infant and young child feeding practices with cognitive development at 10-12 years: a birth cohort in rural Western China. Br J Nutr 2020;123(7):768-79. doi: https:// dx.doi.org/10.1017/S0007114519003271

17. Paslakis G, Dimitropoulos G, Katzman DK. A call to action to address COVID-19-induced global food insecurity to prevent hunger, malnutrition, and eating pathology. Nutr Rev 2021;79(1):114-16. doi: https://dx.doi.org/10.1093/nutrit/ nuaa069

18. Fore H.H, Dongyu Q, Beasley DM, Ghebreyesus TA. Child malnutrition and COVID-19: the time to act is now. Lancet 2020;396:517-18. doi: https://dx.doi.org/10.1016/S01406736(20)31648-2

19. World Health Organization. Mental health and psychosocial considerations during the COVID-19 outbreak. 2020 [documento na internet]. Genebra: WHO; 2020 [atualizado em 18 de março de 2020; citado em 10 de agosto de 2020]. Disponível em: https://apps.who.int/iris/handle/10665/331490

20. Wang G, Zhang Y, Zhao J, Zhang J, Jiang F. Mitigate the effects of home confinement on children during the COVID-19

Recebido em: 21/12/2020 outbreak. Lancet 2020;395(10228):945-47. doi: https://dx.doi. Aceito em:10/06/2021

org/10.1016/S0140-6736(20)30547-X

Como citar: PAZ, Ingre et al. Desafios na atenção à saúde da criança durante a pandemia de Covid-19: o que não deve ser esquecido. Revista Interdisciplinar de Promoção da Saúde, Santa Cruz do Sul, v. 3, n. 4, out 2020. ISSN 2595-3664. Disponível em: <https://online.unisc.br/seer/index.php/ripsunisc/article/view/16112>. Acesso em: 01 out. 2020. doi:https://doi. org/10.17058/rips.v3i4.16112 